\title{
The Property of a Special Type of Exponential Spline Function
}

\author{
Ge Yang \\ North China University of Technology, Beijing, China \\ Email:672322588@qq.com
}

Received 12 October 2015; accepted 14 November 2015; published 17 November 2015

Copyright (C) 2015 by author and Scientific Research Publishing Inc.

This work is licensed under the Creative Commons Attribution International License (CC BY).

http://creativecommons.org/licenses/by/4.0/

c) (i) Open Access

\begin{abstract}
Approximation theory experienced a long term history. Since 50' last century, the rise of spline function as well as the advance of calculation promotes the growth of classical approximation theory and makes them develop a profound theory in maths, and application values have shown among the field of scientific calculation and engineering technology and etc. At present, the study of spline function had made a great progress and had a lot of fruits, as for that, the reader could look up the book [1] or [2]. Nevertheless, the research staff pays less attention to exponential spline function, since polynomial spline function is a special case of that, so it is much essential and meaningful for one to explore the nature of exponential spline function.
\end{abstract}

\section{Keywords}

Exponential Spline Function, Interpolation, Error Estimation

\section{Introduction}

At the beginning, we introduce the definition of exponential spline function. From literature [3], we could learn the definition: if function $S(t)$ satisfies equation $L[S(t)]=\sum_{k} c_{k} \delta\left(t-t_{k}\right)$, we describe it as exponential spline function, where $L$ is a differential operator $L f(t)=D^{n+1} f+a_{n} D^{n} f+\cdots+a_{0} D^{0} f$. Here, $a_{i} \in R$ $(0 \leq i \leq n)$ are constant coefficient and $D^{k}$ represent $k$ th-order derivative. By this definition, we learn that $S(t)$ exists continuous derivative $n-1$ and in each interval $S(t)$ is linear combination of $\left\{t^{k-1} \mathrm{e}^{\alpha_{(m)^{t}}}\right\}_{m=1, \cdots, N_{d} ; k=1, \cdots, k_{(m)}}\left(\sum_{m=1}^{N_{d}} k_{(m)}=n+1\right)$, where the $\alpha_{(m)}$ 's are the $N_{d}$ distinct roots of characteristic polynomial and $\alpha_{(m)}$ is of order $k_{(m)}$. As exists a single root 0 for characteristic polynomial, $S(t)$ is polynomial 
spline function. Next we will deal with the case of there being unique real root.

\section{Main Result}

\section{Theorem 1:}

If the differential operator's characteristic polynomial is $L(s)=(s-\alpha)^{n+1} \quad(\alpha \in R)$, where $\alpha$ is a root of multiplicity $n+1$. Then the expression for exponential spline function of this special case is

$$
S(x)=S_{0}(x)+\sum_{j=1}^{N} c_{j} \mathrm{e}^{\alpha x}\left(x-x_{j}\right)_{+}^{n} \quad x \in[a, b] .
$$

Proof:

Let $S(x)$ be on interval $\left[x_{i}, x_{i+1}\right](i=0,1, \cdots, N), S(x)=S_{i}(x) \in \operatorname{span}\left\{\mathrm{e}^{\alpha x}, \mathrm{e}^{\alpha x} x, \cdots, \mathrm{e}^{\alpha x} x^{n}\right\}$ Suppose $\eta(x)=S_{1}(x)-S_{0}(x)$ And we have $\mathrm{e}^{-\alpha x} \eta(x)=\mathrm{e}^{-\alpha x}\left(S_{1}(x)-S_{0}(x)\right)$

$$
\left[\mathrm{e}^{-\alpha x} \eta(x)\right]^{(i)}=\sum_{k=0}^{i} C_{i}^{k}\left(\mathrm{e}^{-\alpha x}\right)^{(k)} \eta^{(i-k)}(x) \quad(i \leq n-1)
$$

Since there exists order $n-1$ continuous derivatives for $S(x)$, Hence

$$
\eta^{(i)}\left(x_{1}\right)=0 \quad(i=0,1, \cdots, n-1)
$$

So that $\left.\left[\mathrm{e}^{-\alpha x} \eta(x)\right]^{(i)}\right|_{x=x_{1}}=0(0 \leq i \leq n-1)$

Furthermore, $\mathrm{e}^{-\alpha x} \eta(x)$ is polynomial of $n$th degrees.

$$
\text { Therefore } \mathrm{e}^{-\alpha x} \eta(x)=c_{1}\left(x-x_{1}\right)^{n} .
$$$$
\text { We get } S_{1}(x)=S_{0}(x)+c_{1} \mathrm{e}^{\alpha x}\left(x-x_{1}\right)^{n} \text {, }
$$

$$
\text { put } x_{+}=\left\{\begin{array}{ll}
x & x \geq 0 \\
0 & x<0
\end{array}\right. \text {. }
$$

In terms of this idea, we obtain $S(x)=S_{0}(x)+\sum_{j=1}^{N} c_{j} \mathrm{e}^{\alpha x}\left(x-x_{j}\right)_{+}^{n} x \in[a, b]$.

Theorem 2: The dimension of the exponential spline function space is $n+N+1$.

Proof:

Suppose $S(x)=p(x)+\sum_{j=1}^{N} c_{j} \mathrm{e}^{\alpha x}\left(x-x_{j}\right)_{+}^{n}, \quad p(x) \in \operatorname{span}\left\{\mathrm{e}^{\alpha x}, \mathrm{e}^{\alpha x} x, \cdots, \mathrm{e}^{\alpha x} x^{n}\right\}$

We have $\left(S_{i+1}(x)-S_{i}(x)\right)^{(m)}=c_{i} \sum_{k=0}^{m} C_{m}^{k}\left(\mathrm{e}^{\alpha x}\right)^{(k)}\left[\left(x-x_{i}\right)^{n}\right]^{(m-k)}(0 \leq m \leq n-1)$

$$
\text { Since }\left.\left[\left(x-x_{i}\right)^{n}\right]^{(m-k)}\right|_{x=x_{i}}=0
$$

So that $S^{(m)}(x)$ is continuous at the knot $x_{i}(m=0, \cdots, n-1)$, hence $S(x)$ has order $n-1$ continuous derivatives on interval $[a, b]$.

When characteristic polynomial has single real root, the linear space can be written as

$$
\operatorname{span}\left\{\mathrm{e}^{\alpha x}, \mathrm{e}^{\alpha x} x, \cdots, \mathrm{e}^{\alpha x} x^{n}, \mathrm{e}^{\alpha x}\left(x-x_{1}\right)_{+}^{n}, \cdots, \mathrm{e}^{\alpha x}\left(x-x_{N}\right)_{+}^{n}\right\}
$$

Next we prove that $\mathrm{e}^{\alpha x}, \mathrm{e}^{\alpha x} x, \cdots, \mathrm{e}^{\alpha x} x^{n}, \mathrm{e}^{\alpha x}\left(x-x_{1}\right)_{+}^{n}, \cdots, \mathrm{e}^{\alpha x}\left(x-x_{N}\right)_{+}^{n}$ is linearly independent

Set $\sum_{i=0}^{n} c_{i} \mathrm{e}^{\alpha x} x^{i}+\sum_{i=1}^{N} \alpha_{i} \mathrm{e}^{\alpha x}\left(x-x_{i}\right)_{+}^{n}=0$. On the interval $\left[x_{0}, x_{1}\right]$, above equation become $\sum_{i=0}^{n} c_{i} \mathrm{e}^{\alpha x} x^{i}=0$, we 
have $c_{i}=0(i=0,1, \cdots, n)$ On the interval $\left[x_{1}, x_{2}\right]$, we can get $\alpha_{1} \mathrm{e}^{\alpha x}\left(x-x_{1}\right)_{+}^{n}=0$, so that $\alpha_{1}=0$, For the interval $\left[x_{i}, x_{i+1}\right]$, By means of the same technique, we can obtain $\alpha_{i}=0$, hence $\mathrm{e}^{\alpha x}, \mathrm{e}^{\alpha x} x, \cdots, \mathrm{e}^{\alpha x} x^{n}, \mathrm{e}^{\alpha x}\left(x-x_{1}\right)_{+}^{n}, \cdots, \mathrm{e}^{\alpha x}\left(x-x_{N}\right)_{+}^{n}$ is linearly independent. So that we conclude $\operatorname{dim} S=n+N+1$. According to theorem 1. 4. 23 of the book [4], we can prove next conclusion is true.

Corollary: There exists the $S(x)$ for every $f$ belonging to $L^{p}[a, b]$, such that

$$
\|f(x)-S(x)\|_{p}=\min _{s(x) \in S}\|f(x)-s(x)\|_{p}
$$

Theorem 3: If condition of interpolation and boundary satisfy:

$$
\left\{\begin{array}{l}
S\left(x_{i}\right)=f\left(x_{i}\right) \quad i=0,1, \cdots, N+1 \\
S^{\prime}(a)=f^{\prime}(a) \quad S^{\prime}(b)=f^{\prime}(b)
\end{array}\right.
$$

then there exist the $3^{\text {rd }}$ degree exponential spline function satisfied with condition. And we have formula of error evaluation

$$
\|f(x)-S(x)\|_{\infty} \leq c_{0} \mathrm{e}^{|\alpha|(b-a+4)} M h^{4}\left(\text { where } c_{0}=\frac{5}{384}, M=\max _{0 \leq i \leq 4}\left\|f^{(i)}\right\|_{\infty}\right)
$$

Proof:

Suppose $p(x)$ is $3^{\text {rd }}$ degree polynomial spline function, let $S(x)=\mathrm{e}^{\alpha x} p(x)$

Hence $S^{\prime}(x)=\alpha \mathrm{e}^{\alpha x} p(x)+\mathrm{e}^{\alpha x} p^{\prime}(x)$

Both of them can be denoted by: $\left(\begin{array}{l}S(x) \\ S^{\prime}(x)\end{array}\right)=\mathrm{e}^{\alpha x}\left(\begin{array}{cc}1 & 0 \\ \alpha & 1\end{array}\right)\left(\begin{array}{l}p(x) \\ p^{\prime}(x)\end{array}\right), \quad A=\left(\begin{array}{ll}1 & 0 \\ \alpha & 1\end{array}\right),|A| \neq 0$, so that $A$ is invertible matrix. $\quad A^{-1}=\left(\begin{array}{cc}1 & 0 \\ -\alpha & 1\end{array}\right)$

$$
\text { This lead to }\left(\begin{array}{l}
p(x) \\
p^{\prime}(x)
\end{array}\right)=\mathrm{e}^{-\alpha x}\left(\begin{array}{cc}
1 & 0 \\
-\alpha & 1
\end{array}\right)\left(\begin{array}{l}
S(x) \\
S^{\prime}(x)
\end{array}\right)
$$

Since $p(x) \in C^{2}[a, b]$, hence $S(x) \in C^{2}[a, b]$, we can get $S(x)$ is exponential spline function.

If boundary condition is $S^{\prime}(a)=f^{\prime}(a), S^{\prime}(b)=f^{\prime}(b)$, by matrix relation (2), let $p^{\prime}(a)=\mathrm{e}^{-\alpha a}\left(-\alpha f(a)+f^{\prime}(a)\right)$ and $p^{\prime}(b)=\mathrm{e}^{-\alpha b}\left(-\alpha f(b)+f^{\prime}(b)\right)$

Since one of $3^{\text {rd }}$ degree polynomial spline function meet the constraint of interpolation $p\left(x_{i}\right)=\mathrm{e}^{-\alpha x_{i}} f\left(x_{i}\right)$, boundary condition is $p^{\prime}(a)$ and $p^{\prime}(b)$.

So that exponential spline function satisfied with condition (1) exists. That is $\mathrm{e}^{\alpha x} p(x)$.

Next we prove formula of error evaluation. Suppose $f(x) \in C^{4}[a, b], S(x)$ is $3^{\text {rd }}$ degree exponential spline function satisfied with condition (1).

Let $S(x)=\mathrm{e}^{\alpha x} p(x)$ (where $p(x)$ is $3^{\text {rd }}$ degree polynomial spline function)

$$
\begin{aligned}
\|f(x)-S(x)\|_{\infty} & =\left\|f(x)-\mathrm{e}^{\alpha x} p(x)\right\|_{\infty}=\left\|\mathrm{e}^{\alpha x}\left(\mathrm{e}^{-\alpha x} f(x)-p(x)\right)\right\|_{\infty} \\
& \leq\left\|\mathrm{e}^{\alpha x}\right\|_{\infty}\left\|\mathrm{e}^{-\alpha x} f(x)-p(x)\right\|_{\infty}
\end{aligned}
$$

Since $p\left(x_{i}\right)=\mathrm{e}^{-\alpha x_{i}} f\left(x_{i}\right)$

$$
\begin{aligned}
p^{\prime}(a)=-\alpha \mathrm{e}^{-\alpha a} S(a)+\mathrm{e}^{-\alpha a} S^{\prime}(a) & =-\alpha \mathrm{e}^{-\alpha a} f(a)+\mathrm{e}^{-\alpha a} f^{\prime}(a)=\left.\left(\mathrm{e}^{-\alpha x} f(x)\right)^{\prime}\right|_{x=a} \\
p^{\prime}(b) & =\left.\left(\mathrm{e}^{-\alpha x} f(x)\right)^{\prime}\right|_{x=b}
\end{aligned}
$$

By formula of error evaluation for $3^{\text {rd }}$ degree polynomial spline function, we can have

$$
\left\|\mathrm{e}^{-\alpha x} f(x)-p(x)\right\|_{\infty} \leq c_{0}\left\|\left(\mathrm{e}^{-\alpha x} f(x)\right)^{(4)}\right\|_{\infty} h^{4}
$$




$$
\left\|\left(\mathrm{e}^{-\alpha x} f(x)\right)^{(4)}\right\|_{\infty}=\left\|\sum_{k=0}^{4} C_{4}^{k}\left(\mathrm{e}^{-\alpha x}\right)^{(4-k)} f^{(k)}\right\|_{\infty}=\left\|\sum_{k=0}^{4} C_{4}^{k}(-\alpha)^{4-k} \mathrm{e}^{-\alpha x} f^{(k)}\right\|_{\infty}
$$

In terms of book [5], we have

$$
\begin{gathered}
\left\|\sum_{k=0}^{4} C_{4}^{k}(-\alpha)^{4-k} \mathrm{e}^{-\alpha x} f^{(k)}\right\|_{\infty} \leq\left\|\mathrm{e}^{-\alpha x}\right\|_{\infty} M \sum_{k=0}^{4} C_{4}^{k}|\alpha|^{4-k}=\left\|\mathrm{e}^{-\alpha x}\right\|_{\infty} M(1+|\alpha|)^{4} \\
\text { Since } 1+x \leq \mathrm{e}^{x}(x \geq 0) \\
\text { Hence }(1+|\alpha|)^{4} \leq \mathrm{e}^{4|\alpha|} \\
\text { Furthermore }\left\|\mathrm{e}^{\alpha x}\right\|_{\infty}\left\|\mathrm{e}^{-\alpha x}\right\|_{\infty}=\mathrm{e}^{|\alpha|(b-a)}
\end{gathered}
$$

By above expressions, we can conclude that

$$
\|f(x)-S(x)\|_{\infty} \leq c_{0} \mathrm{e}^{\mid \alpha(b-a+4)} M h^{4} .
$$

\section{Fund}

Supported partly by National Natural Science Foundation of China (11126140, 11201007) and partly by Beijing Talents Training Program (2011D005002000006) and partly by Science and Technology Development Plan Project of Beijing Education Commission (KM20121000-9013) and partly by Scientific Research Personnel Promotion Plan of North China University of Technology (BJRC201309).

\section{References}

[1] Li, Y.S. (1983) Spline Function and Interpolation. Shanghai Science and Technology Press, Shanghai.

[2] Feng, Y.Y., Zeng, F.L. and Deng, J.S. (2013) Spline Function and Approximation Theory. University of Science and Technology of China, Hefei.

[3] Unser, M. and Blu, T. (2005) Cardinal Exponential Splines: Part I-Theory and Filtering Algorithms. IEEE Transactions on Signal Processing, 53, 1425-1438. http://dx.doi.org/10.1109/TSP.2005.843700

[4] Zhang, G.Q. and Lin, Y.Q. (1987) Lectures on Functional Analysis. Peking University Press, Beijing.

[5] Li, Q.Y., Wang, N.C. and Yi, D.Y. (2008) Numerical Analysis. 5th Edition, Tsinghua University Press, Beijing. 